\title{
O EXCESSO DE GORDURA VISCERAL É UM FATOR DE RISCO PARA FÍSTULA PANCREÁTICA APÓS GASTRECTOMIA TOTAL
}

0 efeito da obesidade na gastrectomia em pacientes com câncer gástrico é controversa. 0 aumento da gordura visceral aumenta a dificuldade técnica na cirurgia abdominal. $O$ índice de massa corpórea (IMC), apesar de muito utilizado, ainda não mostrou suas reais consequências sobre as complicações da cirurgia gástrica. O IMC aumentado nem sempre reflete o grau de gordura visceral, pois o acúmulo de gordura no organismo varia muito de um indivíduo para outro. A gordura visceral pode ser quantificada por uma tomografia de abdômen com cortes ao nível do umbigo utilizando software específico para isso. Este estudo analisou o efeito da gordura visceral abdominal na gastrectomia total e os fatores de risco associados com a formação de fistula pancreática pós-operatória.

Entre fevereiro de 2001 e abril de 2007, 191 pacientes com câncer gástrico foram submetidos a gastrectomia total com linfadenectomia no Centro Médico de Osaka para câncer e doenças cardiovasculares. Calculou-se a área de gordura visceral por TC ao nível do umbigo utilizando-se o "FatScan Software". Os pacientes foram divididos em grupos de alta gordura visceral ( $>$ $100 \mathrm{~cm} 2, \mathrm{n}=52)$ e em baixa gordura visceral $(<100 \mathrm{~cm} 2, \mathrm{n}=$ 139). Também foram subdivididos em dois grupos de acordo com o IMC em alto (IMC $>25, n=47)$ e baixo (IMC $<25, n=$ 144). Todos os dados referentes aos pacientes (Idade, sexo, IMC, gordura visceral, etc), ao tumor (Estadiamento, linfonodos dissecados, etc), dados da cirurgia (perda de sangue, tempo cirúrgico, etc) e complicações pós-operatórias sofreram análise uni e multivariada, de acordo com o IMC e o acúmulo de gordura visceral.

Os resultados mostraram que a idade, o sexo, a profundidade do tumor, o nível de comprometimento linfonodal, o estádio, a esplenectomia, a perda de sangue durante a cirurgia, o tempo de operação, o número de linfonodos dissecados, a infecção da incisão, a fístula de anastomose, abscesso intra-abdominal, 
hemorragia pós-operatória e íleo paralítico não tiveram significância estatística quando comparados os grupos alta gordura visceral com baixa gordura visceral. A única complicação com significância estatística nesses dois grupos foi o de fístula pancreática, que foi maior no grupo alta gordura visceral (20 em 32 pacientes) quando comparado com o grupo baixa gordura visceral (11 em 128 pacientes).

Quando comparados, não foi encontrada significância estatística entre os dados nos grupos com alto IMC e baixo IMC quanto aos quesitos tempo de operação, número de linfonodos dissecados, infecção da incisão, fistula da anastomose, abscesso intra-abdominal, fístula pancreática, hemorragia pós-operatória e íleo paralítico. 0 único aspecto com significância nesses grupos foi a perda sanguínea intraoperatória, que foi maior no grupo alto IMC.

Quando foram comparados os pacientes com e sem fístula pancreática, constatou-se que o IMC nos dois grupos foi semeIhante. No entanto, a área de gordura visceral, a perda sanguínea intraoperatória e a esplenectomia foram maiores no grupo com fistula pancreática $(p<0,0001)$.

Os autores concluíram que o acúmulo de gordura visceral é um melhor indicador para fístula pancreática pós-operatória que o IMC e que a linfadenectomia e a esplenectomia deve ser realizadas com muito cuidado durante a gastrectomia total nos pacientes com excesso de gordura visceral.

\section{Comentário}

Em nossa experiência pessoal já tínhamos constatado a maior dificuldade operatória em cirurgia abdominal em pacientes com grande quantidade de gordura visceral. Tal dificuldade se constata, principalmente quando se realiza a linfadenectomia na gastrectomia por câncer de estômago. Talvez essa seja a explicação do porquê os resultados dos japoneses quanto à linfadenectomia são melhores que os resultados ocidentais, uma vez que os pacientes orientais sabidamente têm um índice de gordura visceral muito inferior quando comparados aos pacientes ocidentais. A nossa experiência em cirurgia para o tratamento da obesidade mórbida também mostrou que pacientes com alto IMC não têm necessariamente aumento proporcional da gordura visceral.

Carlos Alberto Malheiros ${ }^{1}$ ELIAS JIRJOSS ILIAS ${ }^{2}$

1. Chefe do Departamento de Cirurgia da Faculdade de Ciências Médicas da Santa Casa de São Paulo e Chefe da Área V (estômago, duodeno e obesidade mórbida), São Paulo, SP

2. Professor convidado do Departamento de Cirurgia da Faculdade de Ciências Médicas da Santa Casa de São Paulo, São Paulo, SP

Referência

1.Tanaka K, Myiashiro I, Yano M, Kishi K, Mootori M, Seki Y, et al. Accumulation of excess visceral fat is a risk factor for pancreatic fistula formation after total gastrectomy. Ann Surg Oncol. 2009;16:1520-5. 